\title{
Exploring the potential of video technologies for collaboration in emergency medical care. Part I: Information sharing
}

\author{
Diane H. Sonnenwald*, Hanna M. Söderholm \\ Swedish School of Library and Information Science \\ Göteborg University \& University College of Borås, SE-50190 Borås, Sweden \\ E-mail: \{diane.sonnenwald, hanna.maurin\}@hb.se \\ Telephone: +46 (0)334354200 \\ Fax: +46 (0)334354005 \\ James E. Manning, Bruce Cairns \\ School of Medicine, University of North Carolina, Chapel Hill, NC 27599, USA \\ E-mail: \{james_e_manning, bruce_cairns\}@med.unc.edu \\ Telephone: +1 1919664131 \\ Fax: +1 9199663049

\section{Greg Welch, Henry Fuchs} \\ Department of Computer Science, University of North Carolina, Chapel Hill, NC 27599, USA \\ E-mail: \{welch, fuchs\}@cs.unc.edu \\ Telephone: +1 1919621700 \\ Fax: +19199621799 \\ *Author to which all correspondence should be sent.
}

\section{Exploring the potential of video technologies for collaboration in emergency medical care. Part I: Information sharing}

\begin{abstract}
We are investigating the potential of 3D telepresence, or televideo, technology to support collaboration among geographically separated medical personnel in trauma emergency care situations. 3D telepresence technology has the potential to provide richer visual information than current 2D video conferencing techniques. This may be of benefit in diagnosing and treating patients in emergency situations where specialized medical expertise is not locally available. The 3D telepresence technology does not yet exist and there is a need to understand its potential before resources are spent on its development and deployment. This poses a complex challenge. How can we evaluate the potential impact of a technology within complex, dynamic work contexts when the technology does not yet exist? To address this challenge we conducted an


experiment with a post-test, between-subjects design that takes the medical situation and context into account. In the experiment we simulated an emergency medical situation involving practicing paramedics and physicians, collaborating remotely via two conditions: with today's 2D videoconferencing and a 3D telepresence proxy. In this paper we examine information sharing between the attending paramedic and collaborating physician. Postquestionnaire data illustrate that the information provided by the physician was perceived to be more useful by the paramedic in the 3D proxy condition than the 2D condition. However, data pertaining to the quality of interaction and trust between the collaborating physician and paramedic show mixed results. Post-interview data help explain these results.

\section{Introduction}

Trauma is a significant health problem, frequently referred to as the 'hidden epidemic of modern society' because it is responsible for more productive years lost than heart disease, cancer and stroke combined (Meyer, 1998; Coates \& Goode, 2001). 3D telepresence technology, an emergent information and communications technology that may ultimately provide dynamic 3D views of remote scenes (Welch et al., 2005), has the potential to provide richer visual information than current 2D video conferencing techniques and increase a physician's knowledge about a remote patient, the trauma scene, and actions performed by remote medical personnel. Using 3D telepresence technology (also referred to as 3D televideo technology) may provide people in rural, catastrophic and hazardous areas, as well as disabled and elderly people, who cannot be easily transported access to experts at large medical care facilities.

As indicated in the National Academy's report on telemedicine evaluation (Field, 1996), it is of critical importance to examine the acceptability and practicality of technology in medicine. Today only prototype demonstrations of the 3D telepresence technology exist; the technology is not sufficiently developed to be evaluated using standard usability methods. However, research funding agencies would like to know today whether the technology has potential to improve health care in emergency medical care situations. The funding agencies would like to avoid spending millions of dollars developing yet another technology that does not have a positive impact on medical care. This poses a complex challenge. How can we evaluate the potential impact of a technology within complex, dynamic collaborative work contexts when the technology does not yet exist?

To address this challenge we conducted an experimental evaluation that compared collaboration between paramedics and remote physicians under two conditions. One condition utilized high quality, state-of-the-art 2D videoconferencing to support collaboration during a simulated emergency medical situation. The other condition utilized a 3D telepresence proxy because as discussed above, the 3D telepresence technology does not yet exist. In each condition the same emergency 
medical situation was simulated. A professional paramedic was asked to diagnose and treat a trauma victim, and a physician was available to assist the paramedic. In the 2D condition, the paramedic and physician interacted via state-of-the-art bidirectional and remote-controlled 2D video and audio. In the 3D proxy condition, the paramedic and physician were collocated but the physician could not touch anyone or anything in the room. Performance and posttest data were collected and analyzed to compare the impact of technology in each condition.

This paper discusses the results concerning information sharing and collaboration between the paramedics and physicians. If information is not effectively shared between a paramedic and remote physician in emergency situations, patient care may suffer. For example, if a remote physician's view of the patient is obscured the physician may ask questions that the paramedic finds superfluous and a waste of time that could otherwise be spent treating the patient. If a remote physician does not provide information about a procedure in a useful way for the given situation, a paramedic may not be able to perform the procedure correctly or at all. In our study we examined usefulness of information, quality of interaction and trust. Results indicate that the information provided by the consulting physician was perceived to be more useful by the paramedic participating in the 3D proxy condition than the 2D condition. However, the data pertaining to the quality of interaction and trust between the consulting physician and attending paramedic showed mixed results.

In postquestionnaires paramedics reported that their interaction with physicians was less constrained in the 3D proxy condition than in the 2D condition, but not necessarily more accurate. In postinterviews all paramedics reported an initial awkwardness interacting with the physician, but paramedics in the 2D condition also reported a variety of other challenges, including wanting the physician to provide more feedback during the diagnosis and treatment process.

Trust can be operationalized as ten attributes (Butler, 1991): competence, consistency, fairness, integrity, loyalty, openness, availability, promise fulfillment, receptivity, and discreetness. There were only statistically significant differences in postquestionnaire responses regarding competence, promise fulfillment and receptivity, with paramedics in the 3D proxy condition rated the consulting physicians higher with respect to these attributes. Interview data help explain these results. In interviews paramedics discussed how they are required by law to trust physicians, yet in the 2D condition paramedics experienced frustration when the physicians could not clearly see the intricate procedures the paramedic was performing and made unconstructive comments.

In this paper we review relevant related research, and introduce the experiment design. We present findings regarding information sharing based on our quantitative and qualitative data analysis, and discuss implications of these findings. A subsequent paper, "Exploring the potential of video technologies for collaboration in emergency medical care. Part II: Task 
performance”, further explores the potential of video technologies presenting our findings regarding task performance and self-efficacy.

\section{Related research}

\subsection{Telemedicine and emergency health care situations}

Telemedicine is defined as "the use of electronic information and communications technologies to provide and support health care when distance separates the participants” (Field, 1996, p. 1). Telemedicine includes a wide array of applications, ranging from transmission systems for sharing medical data such as X-ray pictures to remote health monitoring systems for elderly and chronically ill individuals, from videoconferencing systems for counseling or consultation between patients and physicians or other medical specialists to video and haptic systems to support remote surgery. The majority of previous research on video and visual technologies in telemedicine focuses on consultation among a patient, a physician and/or other health care personnel, or between physicians at distributed locations in non-emergency situations (e.g., Alem, Hansen \& Li, 2006; Bardram, Bossen \& Thomsen, 2005; Li, Wilson, Stapleton \& Cregan, 2006; Mbarika, 2004).

An emergency situation such as a car accident scene is different from the traditional telemedicine contexts mentioned above. For example, the physical surroundings might be challenging with respect to weather situation, and/or they can be unsafe involving risk of fire or explosions from damaged vehicles. There is often a need for time critical information regarding what has happened, and the type and quantity of injuries the victims have sustained in order to determine what actions and patient treatment are required. When arriving at the scene of an accident, the patient is a paramedic's primary source of information of what actions to take. The paramedic must interpret the accident scene, patients’ symptoms, injuries and physical responses in order to determine appropriate treatment. If the patient is awake and conscious, the paramedic can get additional information by asking the patient questions about symptoms, pain, the accident, patient history, etc. Sometimes the paramedic has a partner or another emergency medical technician (EMT) available to discuss treatment options with, but in multiple victim casualties a paramedic may need to work alone with a patient. Another source of information is a protocol book describing drug dosages and treatment procedures the paramedic is allowed to perform. However, in complex trauma cases or when the paramedic is having difficulty diagnosing the patient, needs advice regarding a procedure that is to be performed, and/or needs legal permission to perform a specific procedure, paramedics must consult with a physician at a hospital or medical facility.

Today when paramedics are in the field away from a hospital or medical center providing emergency care, collaboration, or consultation, with remote physicians occurs via cell phone or radio. During this collaboration, a paramedic 
must quickly and accurately do the following: verbally describe the victim, the accident scene and the victim's symptoms; answer the consulting physician’s questions; discuss treatment options with the physician; monitor the victim's progress; and simultaneously perform complex medical procedures to save the victim's life. All of these activities must often be performed within minutes. An incorrect description and subsequent decision and/or action could result in death, or further complications and a longer recovery time for the patient, or victim. Thus, videoconferencing technologies for information sharing could be beneficial in emergency healthcare situations by providing the consulting physician with a directly transmitted view of the patient and accident scene. This is especially important when there are long transport times to nearest hospital and patients are severely injured and/or in need of immediate care beyond the level that paramedics are authorized to provide or feel comfortable providing.

\subsection{Computer Supported Collaborative Work}

Previous research in computer supported cooperative work (e.g., Dourish, Adler, Bellotti, \& Henderson, 1996; Olson \& Olson, 2000), theory of language (Clark, 1996) and theory of situation awareness (e.g., Sonnenwald, Maglaughlin, \& Whitton, 2004) suggests that the visual space that is shared when working remotely using 2D videoconferencing lacks the richness of collocation and face-to-face interaction. The usefulness of information shared and the quality of interaction during remote collaboration may suffer because multiple and redundant communication channels, implicit cues, and spatial co-references that are difficult to support via technology-mediated communication. This lack of richness can make it more difficult for individuals to understand the meaning of each other's utterances and actions, and more difficult to develop and maintain an accurate contextual, task process, and socio-emotional awareness of the remote situation. These difficulties may impair emergency medical collaboration in telemedicine applications. Yet for some types of work, remote collaboration mediated by technology can be as effective as working face-to-face. For example, Sonnenwald, Maglaughlin and Whitton (2003) show that science students working remotely via collaborative access to a specialized scientific instrument, videoconferencing and shared applications were able to complete a natural science lab experiment as effectively as students working face-to-face.

Another important aspect of computer-supported cooperative work is trust. There are many definitions of trust arising from different disciplinary perspectives. When synthesizing these definitions, Rosseau, Sitkin, Burt and Camerer (1998) found that scholars fundamentally agree that trust is a "psychological state comprising the intention to accept vulnerability based upon positive expectations of the intentions or behavior of another” (p. 395). Trust involves judgments regarding a person’s competence, consistency, fairness, integrity, loyalty, openness, availability, promise fulfillment, receptivity, and/or 
discreetness (Butler, 1991). Trusting another person involves risk, or the probability of loss, and interdependence, or reliance on others. Collaboration of any type is not possible without trust. When individuals believe that a person is not trustworthy, they will chose not to collaborate with that person or, if forced to collaborate, may sabotage that collaboration. Previous research has shown that when work teams are geographically distributed, trust among members is negatively impacted because mechanisms, such as informal face-to-face interactions and observations, that typically are used in building and maintaining trust are not universally present (Handy, 1995; Rocco, Finholt, Hofer, \& Herbsleb, 2001; Jarvenpaa \& Leidner, 1995).

\subsection{Current videoconferencing technologies for health care collaboration}

In the telemedicine literature two problems with respect to 2D videoconferencing technology are repeatedly mentioned: the difficulty associated with obtaining the desired camera views and the lack of depth perception. In emergency situations today physicians must resort to secondary visual cues or verbal clarification from a remote paramedic, which impose additional cognitive loads during time-critical and possibly life-threatening situations compared to the very natural views afforded if the consulting physician were able to "be there” with the patient. Patients also recognize this problem. In a study at Georgetown University Medical Center (Mun, 2000) patients reported that the physician could not always "see” how the patient was "really doing." To address the visibility problem, the use of multiple cameras and camera views has been proposed, e.g., providing a picture-in-a-picture or a large display of multiple views (Sellen, 1992). However switching between multiple disjoint views, as a security guard might with a surveillance system, is not very helpful in time-critical health care situations. For example, Fussel, Setlock and Kraut (2003) found that users had problems knowing which view to focus on when being presented a combination of a head-mounted camera view and a scene-view of a remote location, thus impacting task performance negatively.

With a very large number of cameras and user head tracking, automatic switching based on view position and orientation could help. But the quantity and configuration of cameras necessary to achieve smooth and appropriate switching over a remote emergency scene, as well as the 2D video storage and bandwidth needs, would be impractical. While pan-tilt-zoom cameras can help address this problem, they require additional technical skills, impose an additional cognitive load, and require additional time to adjust (which is difficult in a trauma situation). Hauber, Regenbrecht, Billinghurst and Cockburn (2006) found that feelings of co-presence increased when improving spatiality in 2D videoconferencing by $3 \mathrm{D}$ views, while performance of a collaborative task was better when done via $2 \mathrm{D}$ videoconferencing 
alone. Hauber suggests that the menu-systems and navigation tools used in their particular system seemed to increase the users' cognitive load, thus making it harder to perform the task with the added spatiality (3D view) than via 2D video (Hauber et al., 2006). The use of menu-systems and need for navigation could be extremely impractical in an emergency situation as a remote physician is fully dependent on immediately obtaining the desired view of the patient in order to be able to give advice regarding patient care. There is simply little or no time for navigation in a system or manual manipulation of equipment.

In addition to approaches for getting the desired view of a remote patient as described above, Tachakra states that “impaired depth perception is a significant problem in telemedicine” (Tachakra, 2001, p. 84). Tachakra suggests a controlled camera rotation that "enables the consultant to build a three-dimensional mental image of the object by briefly storing a range of two-dimensional views” (Tachakra, 2001, p. 83). This is not surprising because object occlusion and motion parallax (i.e., changes in the perceived position of an object compared to background objects as we move) are two of the most powerful depth cues. However, it may often not be realistic to perform camera rotation as prescribed by Tachakra in time-critical trauma emergencies in the field. The time taken to rotate a camera and view will reduce the time available for patient care. It could also reduce the number of on-site personnel who can provide treatment to a trauma victim when an on-site person is required to solely, or primarily, focus on rotating the camera. Furthermore, it could be physically very difficult to rotate a camera, e.g., when a victim of a car accident is lying on a hillside along the side of a road. To address these limitations research is being conducted on how 3D telepresence technology could provide depth perception and dynamic views.

\subsection{D Telepresence technology}

The current vision for 3D telepresence, or televideo, technology would provide users with a seamless view of a remote scene, and the ability to virtually point to objects at the remote location (Welch et al., 2005). This view would, ideally, dynamically change as the user (in our case a physician) walks around, changes position, or moves their head. To track their head and corresponding eye gaze, the user would wear a small head-mounted tracking device. The view of the remote scene would then dynamically change in real time corresponding to where in the scene the user's eyes are currently gazing. A digital laser pointer could be used and projected at the remote scene to allow the user to virtually point at but not touch objects and areas in the remote location. Today, these capabilities are being explored using an array of cameras to capture the remote scene and computer algorithms to blend those camera views and present the result to remote users in real time (Welch et al., 2005). Ideally there will be no need for navigation tools that require user input or manipulation such as zoom 
or change the angles of the cameras. The goal is for users to see a 3D representation of the remote scene and be able to dynamically change their viewpoint of that scene in real-time. In trauma and other emergency medical situations it could enable the physician to virtually move around the trauma victim, the paramedic and the equipment at a remote accident scene. It should enable the physician to quickly look at different parts of the victim's body and to look more closely at an ongoing intricate surgical procedure. Only an early prototype system demonstrating components of 3D telepresence technology exists today (Welch et al., 2005).

\subsection{Research Hypotheses}

As discussed above, previous research has shown that the usefulness of information, quality of interaction and trust among team members can be negatively impacted in technology-mediated collaboration. However the goal of 3D telepresence technology is to enable richer interaction than is currently possible in today's 2D videoconferencing technology. Therefore, our hypotheses are:

H1: Paramedics will perceive the usefulness of information provided by a consulting physician greater when collaborating via $3 D$ telepresence technology than when collaborating via $2 D$ videoconferencing.

H2: Paramedics will rate the quality of interaction with a consulting physician higher when collaborating via $3 D$ telepresence technology than when collaborating via $2 D$ videoconferencing.

H3: Paramedics will report greater levels of trust with respect to a consulting physician when collaborating via $3 D$ telepresence technology than when collaborating via $2 D$ videoconferencing.

\section{Research Design}

\subsection{Research Approach}

The theory of information horizons (Sonnenwald, 1999) states that human information behavior, including information seeking, sharing, creation and use, is shaped by and shapes individuals, social networks, situations and contexts. Information behavior emerges from reflections regarding others, self and the environment, in particular, reflections concerning a lack of knowledge. To understand human information behavior we should investigate individual perceptions, relevant social networks, the situation and context in which information behavior occurs. Such investigation is typically done through observations and interviews. 
However investigating information behavior during remote consultation in emergency medical situations has unique challenges, many of which can be attributed to the complex contexts in which emergency situations occur. Patient healthcare priorities, patient privacy, and the dynamics of emergency care in the field make it extremely difficult, if not impossible, to collect observation data in the field. These conditions make observation as a research method impractical.

Interviewing paramedics is also not feasible when investigating collaboration mediated by video-conferencing and 3D telepresence technologies. Today paramedics use radio or cell phones to collaborate with physicians during emergency situations. They do not use videoconferencing and can not use 3D telepresence technology to collaborate. Videoconferencing is not available to paramedics in the field and only limited demonstration prototypes of 3D telepresence exist in computer science research labs. Interviewing paramedics to discuss their perceptions regarding information behavior mediated by technologies they have not used is not a valid research approach.

Because observations and interviews are not feasible we chose to conduct a controlled lab study using a post-test, between-subjects design. However, unlike some controlled lab studies where as much context as possible is purposefully eliminated and artificial situations are created, our study embraces both context and situation. That is, we strove to include as much of the relevant context and situation as possible in the lab study. We realistically simulated an emergency medical situation and had practicing emergency medical physicians and paramedics participate in the simulation under two conditions: collaborating with a physician using state-of-the-art 2D videoconferencing, and collaborating with a physician using a 3D telepresence proxy.

\subsection{Simulation of an emergency medical situation}

In the U.S. paramedics (EMT-P job classification) are typically the first and only medical personnel who travel to the scene of medical emergencies. At the scene their job is to diagnose patients, perform all necessary medical procedures they are legally allowed to perform, and when necessary, transport patients to the nearest available and appropriate medical facility. They typically have no prior knowledge about the patient and limited knowledge about the accident. They often must work quickly and accurately to save lives. We created a simulation of such an emergency medical situation in our lab. In particular, we simulated a car accident scene where a male adult, age 38, was found thrown from a car and moaning.

We used a computerized mannequin, the METI Human Patient Simulator ${ }^{\mathrm{TM}}$, to simulate the trauma patient. This stateof-the-art mannequin can be programmed to act and respond in a life-like manner. For example, its pupils dilute in response to light, its chest rises and falls when breathing, its heart rate, breathing pattern and oxygen blood levels respond 
to drug injections and medical procedures.

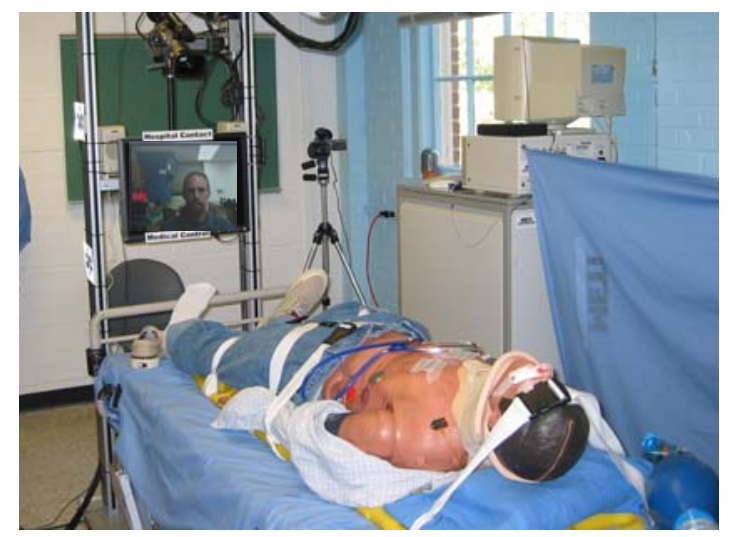

Figure 1. The human patient simulator staged as a car accident victim.

We developed the simulated scenario in collaboration with three physicians with different specialties and subsequently tested and refined the scenario with five paramedics in a pilot study. We used a darkened room with spotlights and a rotating beacon to simulate an accident scene late in the evening with streetlights and the presence of an ambulance (Figure 1). To increase the fidelity we added background sounds that included traffic noises, people's voices and emergency vehicle sirens.

One key aspect of the simulation design was choosing an appropriate trauma emergency medical task for the participating paramedics to perform. The task needed to have a certain level of complexity, consisting of demanding medical decision-making and technical skills. It needed to be a task paramedics were qualified to perform with measurable outcomes, and a task they would seek advice on. In addition the task needed to be socially relevant—an important medical problem.

The task selected was the diagnosis and treatment of a difficult airway, including performing a surgical cricothyrotomy. In a surgical cricothyrotomy an incision is made in the neck, through the skin and the underlying cricothyroid membrane, to allow air to pass to the lungs (American Society of Anesthesiologists Task Force, 2003; Elliot, 1999). Paramedics are expected to be able to manage the difficult airway and perform a surgical cricothyrotomy, yet this is extremely challenging. Even the most experienced physicians in airway management recognize the sense of urgency and anxiety associated with control of the difficult airway because patients without an adequate airway will die within minutes if they do not receive appropriate treatment (Gawande, 2001). The inability to secure an airway is the most common cause of preventable death in the pre-hospital care of injured patients (Bair, Panacek, Wisner, Bales, \& Sakes, 2003). We programmed the mannequin to display a difficult airway that required a cricothyrotomy to be performed in order to save the patient. 


\subsection{Conditions}

\section{Table 1. Summary of each condition}

\begin{tabular}{lcc}
\hline Actor & \multicolumn{2}{c}{ Condition } \\
\hline Study participant & 2D & 3D proxy \\
& 20 paramedics & 20 paramedics \\
Emergency care physician (collaborator) & Via bi-directional video \& audio & Collocated with constraints \\
Mannequin (accident victim) & Collocated & Collocated \\
& & \\
Researcher-observer & Collocated & Collocated \\
Mannequin operator technician & Via audio & Via audio \\
\hline
\end{tabular}

State-of-the-art, high quality 2D videoconferencing was used in the 2D condition (see Table 1.) Three views of the mannequin were provided to the remote consulting physician using digital cameras directly connected to three 21-inch high resolution monitors. One camera was a remote-controlled pan-tilt-zoom camera that the consulting physician could control. All cameras were placed in optimal positions for our particular emergency medical situation. That is, the expert physicians determined the best locations for the three cameras to enable effective observation of the diagnosis and management of a difficult airway on the mannequin.

The consulting physician also had a full screen view of the mannequin’s patient monitor showing the mannequin's heart rate, blood pressure and blood oxygen saturation rates in real time. The consulting physician observed the patient monitor and camera views in a custom built work station (Figure 2). In addition, the paramedic also had a 2D video view of the consulting physician during the simulation. This view was located at the end of the mannequin (Figure 1).

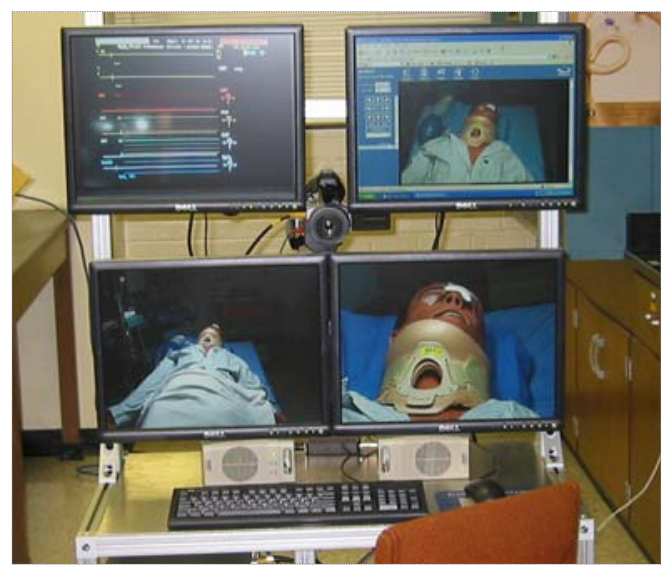

Figure 2. Consulting physician's view in the 2D condition.

As mentioned earlier, the 3D telepresence technology is not yet sufficiently developed to allow us to use it even under ideal lab conditions. Therefore we designed a 3D proxy, or surrogate. For the 3D proxy condition, the consulting physician 
was physically present in the same room as the mannequin and paramedic. The physician was allowed to freely move around in the room. However, the physician could not touch anything in the room and could only point to things using a laser pointer.

This simulates the current vision and technical goals for 3D telepresence technology (Welch et al., 2005). In this vision, remote consulting physicians will wear a small head-mounted tracking device that tracks their head movement and corresponding eye gaze. The physician's view of the accident scene will dynamically change in real time corresponding to where their eyes are currently gazing. (Alternatively the remote physician might use a hand-held tracking device to change their view of the accident scene.) This may be accomplished by using an array of cameras to capture the remote scene and computer algorithms to blend those camera views and present the result to remote physicians in real time. The goal is for remote physicians to see a 3D representation of the accident scene and be able to dynamically change their viewpoint of that scene. A remote physician will also be able to interact with the remote scene through a laser pointer that was displayed in the physician's view and at the accident scene.

Two emergency care physicians acted as the consulting physicians in the 2D and 3D proxy conditions, with each physician participating in an equal number of sessions across the two conditions. To help reduce the potential impact of any individual differences between the physicians, an interaction script was used by the physicians. The script consisted of appropriate, constructive phrasing of responses to typical questions and advice to give the paramedic at certain times during the scenario. The main reason for using the script was to minimize individual differences between the physicians regarding tone of voice and advice given to paramedics. The script was based on actual physician-paramedic interaction observed during a pilot study and was developed in collaboration with the physicians.

Social facilitation theory (Bradner \& Mark, 2001; Cottrell, Wack, Sekerak \& Rittle, 1968) suggests that an individual’s performance is affected by the physically (collocated) and virtual (remote) presence of an audience, in the sense that a person being observed by an audience will perform easier tasks better and more difficult tasks worse. This effect occurs when one person is present. If two or more people are in the audience, either physically or virtually, the impact is the same as if only one person is in the audience. That is, social facilitation theory indicates that the presence of an additional audience member, physically and virtually, would have no additional impact on a person’s performance.

In our experiment during all experiment sessions for all conditions, the physical (collocated) audience always consisted of a researcher-observer, and the virtual audience always consisted of an expert mannequin operator observing the study 
participant from an adjacent room. All paramedics were aware of the presence of both the physical and virtual audience. Thus all paramedics in the study had both a physical and virtual audience each consisting of at least one person. Social facilitation theory indicates that the physical presence of an additional physical or virtual audience member, i.e., the physician in the 3D proxy and 2D conditions, would have no additional impact on paramedics’ performance.

\subsection{Study participants}

To determine the optimal number of sessions to be conducted, we reviewed the literature for similar studies and found that 10 to 20 sessions per condition was common (e.g., Gale, 1998). It was not possible to perform a power analysis to determine the actual number needed to produce statistically significant results before conducting the experiment because we did not have an estimate for the size of the effect, or differences, between conditions. We conducted 20 sessions per each condition.

The forty participants, 30 males and 10 females, were all professional paramedics working in southeastern U.S. They averaged over 7 years of paramedic experience, with a range of 1 to 24 years of experience as a certified trained paramedic. Most participants had also worked as an emergency medical technician before qualifying as a paramedic, and thus the participants had an average of 11.7 years of emergency services experience overall. A stratified random assignment approach was used to assign participants to conditions. That is, participants were randomly assigned across conditions, with equal distribution of gender and years of experience across both conditions. In the 2D and 3D proxy conditions five and six paramedics, respectively, had met the physician before, a situation that mirrors the daily work of paramedics. Paramedics usually have one or two specific hospitals where they take patients on a regular basis and where they know the some of the physicians in the emergency room. At other times, patients need to be transported to other hospitals or facilities where the paramedic does not know or has not met the physicians before.

\subsection{Experiment session protocol}

Upon arrival at our facility, each paramedic learned about the overall purpose of our research, the format of each experiment session and their rights as study participants. Each paramedic was given a study consent form to read, discuss and sign. Next, each paramedic received an introduction to the simulation, participated in our medical simulation and completed a post-test questionnaire and interview.

In the introduction to the simulation, participants were shown the video equipment and camera views, and given a hands-on tutorial to the mannequin, time to make themselves familiar with the medical equipment available for diagnosing and treating the patient, and background information about the simulated car accident and patient. The background 
information we provided is normally available to paramedics at accident scenes from first responders, e.g., police who first arrive at an accident scene and call for medical assistance. Each session was attended by an observer who was always collocated with the paramedic and mannequin. The mannequin technician operated the mannequin from an adjacent room and was available for questions regarding the patient simulator before and after the session, as well as during the actual simulation (through an open microphone).

The sessions took an average of 11 minutes, with a range of 6 to 23 minutes. During each session, the paramedic needed to diagnose and treat the victim (mannequin) as discussed earlier. Each session was videorecorded using four cameras that captured paramedic's actions on and surrounding the mannequin and the mannequin’s medical monitor output (heart rate, oxygen saturation levels).

After each session each paramedic completed a postquestionnaire to report their perceptions of satisfaction, selfefficacy, communication and trust with the physician (for the 2D and 3D proxy conditions). Each paramedic also participated in an open-ended interview that was audio-recorded and transcribed. Topics discussed during the postinterview included current work practices, interaction with the physician, and impressions of 2D videoconferencing and 3D technology.

\section{Research Validity and Limitations}

\subsection{Ecological validity}

Table 2. Ecological validity

\begin{tabular}{|c|c|c|c|c|}
\hline & \multicolumn{4}{|c|}{ Questionnaire Responses* } \\
\hline & \multicolumn{2}{|c|}{ 2D Condition } & \multicolumn{2}{|c|}{ 3D Proxy Condition } \\
\hline & Mean & SD & Mean & SD \\
\hline I was absorbed intensely in the activity & 6.20 & .77 & 6.26 & .81 \\
\hline I concentrated fully on the activity & 6.30 & .80 & 6.35 & .75 \\
\hline The simulation was realistic & 5.75 & 1.29 & 6.20 & .83 \\
\hline $\begin{array}{l}\text { Most people would agree on how to treat the patient in the } \\
\text { simulated scenario }\end{array}$ & 5.80 & .83 & 6.30 & .80 \\
\hline Overall average scores per condition & 6.01 & .67 & 6.28 & .46 \\
\hline
\end{tabular}

* Response Scale: 1 (strongly disagree) to 7 (strongly agree)

Smith and Gaba (2000) define a simulation as "the artificial replication of sufficient elements of a real-world domain to achieve a specified goal” (2000, p.1) and the level of accuracy of which the simulation reproduces the domain can be referred to as fidelity. To investigate the participants’ perspective regarding the ecological validity of our simulation, i.e., how closely the simulation mirrors real world conditions, we included several questions in the postquestionnaire about the 
fidelity of the simulation and the participants’ engagement during the simulation. The highest possible fidelity attained would be a simulation so precise that the participants in the simulation not could distinguish it from the real thing (Smith $\&$ Gaba, 2000).

Overall, the participants reported that they concentrated on the activity, had sufficient training, and believed the simulation and medical task were realistic and doable (Table 2.) A Mann-Whitney $U$-test ${ }^{1}$ yielded no statistically significant differences in perceptions of ecological validity due to condition, at the 0.05 level of significance. In postinterviews, participants confirmed their questionnaire responses, saying:

It felt real. [I was] absolutely absorbed. Absolutely.

It was like life.

\subsection{Common ground and internal validity}

Table 3. Common ground

\begin{tabular}{|c|c|c|c|c|}
\hline & \multicolumn{4}{|c|}{ Questionnaire Responses* } \\
\hline & \multicolumn{2}{|c|}{ 2D Condition } & \multicolumn{2}{|c|}{ 3D Proxy Condition } \\
\hline & Mean & SD & Mean & SD \\
\hline $\begin{array}{l}\text { I was familiar with the terminology used by the physician } \\
\text { (shared language) }\end{array}$ & 6.79 & .54 & 6.70 & .57 \\
\hline $\begin{array}{l}\text { I could understand completely what the physician meant when he was } \\
\text { talking (shared language) }\end{array}$ & 6.80 & .52 & 6.85 & .49 \\
\hline $\begin{array}{l}\text { It felt that we were communicating on the same 'wavelength' } \\
\text { (shared language) }\end{array}$ & 6.60 & .50 & 6.75 & .44 \\
\hline I feel that the physician and I care about the same issues (shared goals) & 6.70 & .57 & 6.90 & .45 \\
\hline The physician's goals are consistent and compatible with my own goals & 6.75 & .55 & 6.85 & .49 \\
\hline
\end{tabular}
(shared goals)

* Response Scale: 1 (strongly disagree) to 7 (strongly agree)

Common ground is the information and beliefs that a group (of two or more) have in common, and their awareness that the group has this information and beliefs in common (Clark, 1996; Olson \& Olson, 2000). Previous research, e.g., Olson and Olson (2000) and Carroll, Rosson, Convertino and Ganoe (2006), suggest that common ground is essential for successful collaboration. Internal validity can be threatened if there was no common ground among participating paramedics and physicians regarding emergency medical care. Shared language, shared goals and shared vision are three measures of

\footnotetext{
${ }^{1}$ When testing for differences between independent groups and the data are ordinal and/or may not be normally distributed, nonparametric tests, such as the Mann-Whitney $U$ test, are recommended over parametric tests, such as the ANOVA or t-test. A disadvantage of nonparametric tests is that they have lower statistical power compared to their parametric counterparts, i.e., they are less likely to find a significant difference when there actually is one, especially for small sample sizes. The Mann-Whitney $U$ test we use is the nonparametric counterpart of the parametric t-test (Vaughan, 2001), and we found that this test, ANOVA, and t-tests yield similar statistical significant results for all data reported in this paper.
} 
common ground. To investigate paramedics’ perceptions regarding common ground with physicians, we asked paramedics three questions regarding shared language and two questions regarding shared goals. The questions are based on previous survey questions used by Levin, Cross and Abrams (2002), Levin and Cross (2004), and Smith and Barclay (1997).

Paramedics' responses regarding common ground are shown in Table 3. A Mann-Whitney U-test yielded no statistically significant differences in perceptions of common ground due to condition, at the 0.05 level of significance. Paramedics and physicians equally shared a common language and goals across both conditions.

\subsection{Limitations}

Although our design demonstrates a high degree of validity, it nonetheless has several limitations. One limitation of this study is its post-test, between-subjects design. Alternative experimental designs include a repeated measures design and a Solomon four-group design (Robson, 2002; Shadish, Cook \& Campbell, 2002). In a repeated measures, or within-subjects, design each study participant performs the task under all conditions. This eliminates any potential bias across conditions due to individual differences. However, it introduces a bias due to order of conditions. Study participants typically perform a task better a second time irrespective of the condition. A solution is to vary the order of the conditions and tasks in each condition. However, study participants also learn during each task session (irrespective of the condition and task) and this learning transfers to, and impacts, the second task session. A Solomon four-group design in which additional groups of study participants perform different tasks under each condition in a different order is needed to isolate such learning effects. Greater resources, in terms of numbers of study participants, time and materials, are needed to conduct a Solomon fourgroup experiment. Thus, we used a stratified random assignment approach and a sufficiently large number of study participants to negate potential bias due to individual differences.

A second limitation is the use of a 3D proxy in the evaluation instead of actual 3D telepresence technology. It is possible that the actual physical presence of the consulting physician in the 3D proxy condition had a positive impact on the results that will disappear in 3D telepresence technology. Even a virtual 3D hologram of a remote consulting physician shown to a paramedic (as shown in science fiction films) may not be as effective as a face-to-face consultation. Furthermore, if 3D telepresence technology introduces time delays during transmission and tracking the physician's view, task performance may be negatively impacted. However, if we are to evaluate the potential of a technology that is extremely resourceintensive to develop, some technology proxies or surrogates will be necessary. Our approach, i.e., using a proxy, is similar to Wizard of Oz studies, in which a human simulates the role of an ideal computer (e.g., Höysniemi, Hämäläinen \& Turkki, 
2004). In both approaches simulation is used to evaluate the potential of technology not yet developed and an ideal computer system is assumed. Further research evaluating 3D telepresence technology is needed when prototypes become available.

A third limitation is that we have not yet analyzed the interaction between the paramedics and physician. Such an analysis could discover differences and similarities between the information sharing processes in each condition, and increase our understanding of the information sharing process. We leave this analysis for future work, and focus on six other measures regarding information sharing in this paper.

\section{Results}

\subsection{Usefulness of Information}

\subsubsection{Postquestionnaire results}

Building on previous research by Levin and Cross (2004), usefulness of information was measured through five items on the post-questionnaire that asked to what extent the information received from the consulting physician helped or hindered key aspects of diagnosing and treating the victim in the simulation and future trauma situations. A Likert scale was used for each question response. The scale included seven response options, including: 1 (very negatively); 2 (negatively); 3 (somewhat negatively); 4 (neither positively nor negatively); 5 (somewhat positively); 6 (positively); and, 7 (very positively).

Table 4. Usefulness of information

How did the information/advice you received from the physician contribute to:

Questionnaire Responses*

\begin{tabular}{cccccc}
\hline \multicolumn{2}{c}{ 2D Condition } & \multicolumn{2}{c}{$\begin{array}{c}\text { 3D Proxy } \\
\text { Condition }\end{array}$} & \multicolumn{2}{c}{$\begin{array}{c}\text { Mann-Whitney } \boldsymbol{U} \\
\text { Results }\end{array}$} \\
Mean & SD & Mean & SD & U & $\boldsymbol{p}$ (2-tailed) \\
\hline 5.68 & 1.11 & 6.41 & .80 & 99.00 &. $\mathbf{0 3 5}$ \\
6.00 & .82 & 6.16 & 1.02 & 128.00 & .394 \\
6.30 & .87 & 6.85 & .37 & 125.50 & $\mathbf{. 0 1 4}$ \\
5.89 & 1.15 & 6.84 & .38 & 122.00 & $\mathbf{. 0 0 2}$ \\
6.35 & .81 & 6.84 & .38 & 84.00 & $\mathbf{. 0 2 1}$ \\
6.02 & .73 & 6.63 & .33 & 11.53 & $\mathbf{. 0 0 2}$ \\
\hline
\end{tabular}

(1) Diagnosing the victim

(2) Intubating the victim

(3) Performing a cricothyrotomy on the victim

(4) Increasing your knowledge about managing a difficult airway

(5) Enhancing your future performance in similar situations

Overall mean score per condition

* Response Scale: 0 (did not receive information about this; treated as a missing value), 1 (very negatively) to 7 (very positively)

The data were analyzed using Mann-Whitney $U$-test for comparing scores between conditions. This analysis shows that participants believed that the information received from the consulting physician in the 3D proxy condition was significantly (at the $p<=.05$ level) more useful than most information received in the 2D condition (Table 4). In particular, 
participants' responses indicate that information provided regarding diagnosing and treating the current and future trauma victims was more useful in the 3D proxy condition. There is no statistically significant difference regarding information provided about intubation, and we suspect this is because intubation is a task frequently performed by paramedics and thus they require little or no advice regarding this task.

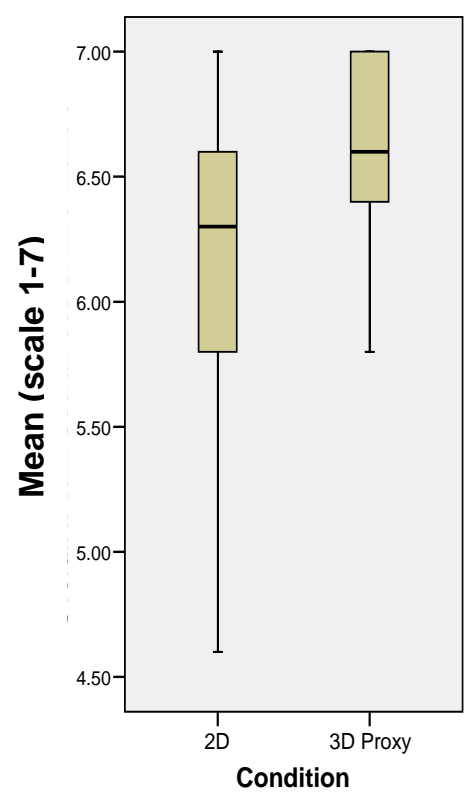

Figure 3. Variance differences in usefulness of information overall.

For all questionnaire items except item 2 (intubating the victim) responses from paramedics in the 2D condition show a greater variance and larger standard deviation than responses from the 3D proxy condition. This is also reflected in the overall average response score (Figure 3). A Levene test for equality of variance shows that these differences are statistically significant for items 3, 4, 5 and the overall mean.

Furthermore, in the 2D condition, two of these questions are negatively correlated with years of professional experience. That is, a rank correlation test using Spearman's rho shows statistical significance at $-.482, p=0.031$ for Item 3: Performing a cricothyrotomy on the victim—overall EMS experience, and -.475, p=0.046 for Item 4: Increasing your knowledge about managing a difficult airway_paramedic experience. This implies that when collaborating via 2D videoconferencing paramedics with fewer years of work experience thought they learned more from the physician than the more experienced paramedics did. There were no such correlations for the 3D proxy condition, suggesting that paramedics in the 3D proxy condition, irrespective of prior work experience, were in close agreement regarding the high value of the information provided by the physician. 


\subsubsection{Postinterview results}

Participants in both the 2D and 3D proxy conditions valued their interaction with the consulting physician. Compared to their current way of working, i.e., collaborating with a physician via radio or a cell phone, using video and a 3D proxy provided better patient healthcare and an opportunity for paramedics to improve their healthcare skills. Participants explained:

With him there we got a better airway...and increased the patient's chance of survival. (3D Proxy participant)

If I had been on the scene without a physician...it probably would have taken 1.5 or 2 minutes longer before [the patient]got a surgical airway. (2D participant)

Everything he was telling me was precise. I knew exactly what to look for. He explained the procedure...I learned something. (3D Proxy participant)

It helped me feel more confident already after [this one session.] (2D participant)

Additional advantages emerged from hands-free communication, reduced wait time when contacting physicians, and interaction with physicians outside one’s geographical area. As participants reported:

Obviously it's going to be hard to hold a telephone, or to hold a radio if I'm trying to do something at the same time... It was nice to be able to have both my hands, and still be able to communicate with him. (2D participant)

Let's say I did have to call for orders for the cric, to move away...from the patient and have to get on the radio..., make sure the radio traffic is clear, and [wait for the nurses] to go locate the physician...and have to wait ... listen to the radio, la la la wait wait wait... In a trauma situation you don't always have a lot of time. (3D Proxy participant)

Maybe...there's a new procedure that being done [at one county hospital] and the patient's in [another county]... maybe you could get the [county hospital] physician's opinion. (2D participant)

However changes to the technology and practices used in the field today may provide similar advantages. For example, voice-activated radios and phones used with headsets could provide hands-free communication.

The use of the laser pointer in the 3D proxy condition also aided information sharing. Physicians used the laser pointer frequently to identify the location and size of the required incision and to point to specific pieces of medical equipment that the paramedic needed to use. The paramedics paid attention to the physician’s pointing. They explained:

He was able to physically point to various things so that was an added bonus.

I liked that he was able to point and tell me what goes where and all. 
As Clark (1996) and other research have indicated the ability to point to physical objects facilitates information sharing, and our results are in agreement with this previous research.

\subsection{Quality of Physician-Paramedic Interaction}

\subsubsection{Postquestionnaire results}

Using questions developed by Chidambram and Jones (1993) and Short, Williams and Christie (1976), we asked each paramedic to answer four questions about their interaction with the physician (see Table 5).

\section{Table 5. Physician-paramedic interaction}

\begin{tabular}{lccccccc}
\hline & \multicolumn{3}{c}{ Questionnaire Responses* } \\
\cline { 2 - 8 } $\begin{array}{l}\text { Please indicate on the scale the number that } \\
\text { best describes your interaction with the }\end{array}$ & \multicolumn{2}{c}{ 2D } & \multicolumn{3}{c}{ 3D Proxy } & \multicolumn{2}{c}{ Mann-Whitney U Results } \\
physician: & Mean & SD & Mean & SD & U & $\boldsymbol{p}$ (2-tailed) \\
1. Free (7)...Constrained (1) & 5.50 & 1.28 & 6.60 & .82 & 92.5 & .002 \\
2. Good (7)...Bad (1) & 6.05 & .83 & 6.50 & 1.0 & 123.5 & .023 \\
3. Accurate (7)...Distorted (1) & 6.40 & .68 & 6.70 & .57 & 150.0 & .113 \\
4. Easy (7)...Difficult (1) & 6.15 & .67 & 6.50 & .76 & 140.5 & .079 \\
Overall average scores per condition & 6.03 & .73 & 6.58 & .57 & 112.0 & $\mathbf{. 0 1 4}$ \\
\hline
\end{tabular}

* Response Scale: 1 to 7

The data analysis shows that interaction between the physician and paramedic was statistically significant (at the $p<=$ .05 level) in three dimensions, i.e., free-constrained, good-bad, and overall (Table 5). Participants in the 3D proxy condition evaluated their interaction with the remote physician much freer and better than participants in the 2D condition. In fact, all physician-paramedic interaction in the 3D proxy condition was perceived more positively than in the $2 \mathrm{D}$ condition but these differences are not statistically significant. The difference with respect to the dimension of free-constrained is together with the good-bad dimension so profound, and the other interaction items so consistent, that when combined the differences between interactions in the 2D and 3D conditions is statistically significant.

\subsubsection{Postinterview results}

Participants in the 2D condition reported more challenges interacting with the physician than participants in the 3D proxy condition. In the 2D condition, 15 participants (75\%) described problems with the paramedic-physician communication. In comparison, 7 participants (35\%) in the 3D proxy condition reported problems. Of those 7, six focused on an initial awkwardness in the communication. A 3D proxy participant explained: 
At first I felt like I was being under the gun, that I was being evaluated. Once I got into the simulation and I got to focus on the patient, and the communication was open, and it was free, and it was non-judgmental, I felt very comfortable.

2D participants also reported an initial awkwardness:

When you start [(interacting] ... [there's an] initial ice breaking thing... the thing I like least is ...the initial awkwardness of it.

In addition to an initial awkwardness, 2D participants mentioned other types of challenges with respect to interaction with the physician. Some reported that the physician should be more assertive, more vocal and ask more questions during the diagnosis and treatment process. Others wanted the physician to give more feedback. Still others reported that the physician could not always see what needed to be seen and this made communication difficult, and one participant noted that he needed to change his behavior to compensate for limitations of the technology. 2D participants reported:

[The physician] could have actually been even more vocal...for example, reading what he's seen on the monitor.

I think it would be better if [the physicians] asked you questions. You know, initiated more.

I like feedback ... I didn't get the feedback... more feedback would have been better.

I could have used [some guidance] on [whether] I should go deeper with the scalpel because I wasn't sure.

I had to remember to give [the physician] more information...once you kinda' get into it... [You] realize, ok, I need to interact more and do this a little different.

With one exception ${ }^{2}$, all these challenges were reported only by 2D participants. We found no correlation between reports of these challenges and paramedics' years of professional experience or participating physician. Therefore these challenges appear to be implicitly linked to 2D video. Even under the best conditions (as in our experiment) the use of 2D video for emergency medical consultation will introduce multiple types of interaction problems.

\subsection{Trust}

\subsubsection{Postquestionnaire results}

As mentioned earlier Butler (1991) proposes trust can be measured using ten variables: competence, consistency, fairness, integrity, loyalty, openness, availability, promise fulfillment, receptivity, and discreetness. Each variable represents one component of trust. Butler created and validated an instrument that contained four questions for each variable plus four questions focusing on overall trust. For each aspect, three questions are positively worded and one is negatively worded (e.g., see Thompson, Rafferty \& Thompson, 2003). Subsequent research (Bos, Olson, Gergle, Olson \& Wright, 2002) used 
a shortened version of Butler's instrument that consists of one question for each variable and one for overall trust. We followed this approach, using 10 questions to measure each component of trust suggested by Butler (1991) and one question that focused on overall trust.

Table 6. Trust

\begin{tabular}{|c|c|c|c|c|c|c|}
\hline & \multicolumn{4}{|c|}{ Questionnaire Responses* } & & \\
\hline & \multicolumn{2}{|c|}{ 2D Condition } & \multicolumn{2}{|c|}{$\begin{array}{l}\text { 3D Proxy } \\
\text { Condition }\end{array}$} & \multicolumn{2}{|c|}{$\begin{array}{l}\text { Mann-Whitney } U \\
\text { Results }\end{array}$} \\
\hline & Mean & SD & Mean & SD & $\mathbf{U}$ & $\boldsymbol{p}$ (2-tailed) \\
\hline $\begin{array}{l}\text { The physician acted competently } \\
\text { (competence) }\end{array}$ & 6.80 & .41 & 7.00 & 0 & 160.0 & .037 \\
\hline $\begin{array}{l}\text { From start to finish, the physician behaved in } \\
\text { a consistent manner (consistency) }\end{array}$ & 6.85 & .37 & 6.90 & .31 & 190.0 & .637 \\
\hline The physician treated me fairly (fairness) & 6.90 & .31 & 7.00 & 0 & 180.0 & .152 \\
\hline The physician acted with integrity (integrity) & 6.84 & .38 & 7.00 & 0 & 160.0 & .068 \\
\hline $\begin{array}{l}\text { The physician did not allow other work to } \\
\text { interfere with assisting you (loyalty) }\end{array}$ & 6.90 & .31 & 7.00 & 0 & 180.0 & .152 \\
\hline $\begin{array}{l}\text { If you gave the physician confidential } \\
\text { information, he would keep it confidential } \\
\text { (discreetness) }\end{array}$ & 6.86 & .36 & 6.89 & .32 & 122.0 & .791 \\
\hline $\begin{array}{l}\text { The physician had an open approach to } \\
\text { treating the victim (openness) }\end{array}$ & 6.80 & .41 & 6.85 & .37 & 190.0 & .681 \\
\hline $\begin{array}{l}\text { It was easy to get the physician's attention } \\
\text { (availability) }\end{array}$ & 6.70 & .47 & 6.90 & .31 & 160.0 & .118 \\
\hline $\begin{array}{l}\text { The physician fulfilled his professional } \\
\text { obligations to me and the victim } \\
\text { (promise fulfillment) }\end{array}$ & 6.80 & .41 & 7.00 & 0 & 160.0 & .037 \\
\hline $\begin{array}{l}\text { The physician really listened to me } \\
\text { (receptivity) }\end{array}$ & 6.65 & .49 & 6.95 & .22 & 140.0 & .019 \\
\hline $\begin{array}{l}\text { I feel that the physician can be trusted } \\
\text { (overall trust) }\end{array}$ & 6.65 & .59 & 6.95 & .22 & 149.5 & .038 \\
\hline
\end{tabular}

* Response Scale: 1 (strongly disagree) to 7 (strongly agree)

As shown in Table 6 all participating paramedics trusted the physicians to a high degree; the lowest average response to any question was 6.60 on a 7 point scale. These responses may be influenced by the U.S. healthcare culture and legal practices which assign high status and legal responsibility to physicians. However all responses regarding trust were lower for the $2 \mathrm{D}$ condition. These lower responses were statistically significant (at $p<=0.05$ ) for competence, promise fulfillment, receptivity and overall trust. That is, paramedics participating in the 3D proxy condition felt the collaborating physician acted more competently, fulfilled his obligations to the paramedic and victim to a higher degree, was more receptive to the paramedic and was more trustworthy overall.

\footnotetext{
${ }^{2}$ One 3D proxy participant reported he also wished the physician had been more assertive. 
These results appear to be attributable to inherent differences in the 2D videoconferencing and 3D technology proxy because individual differences with respect to the collaborating physicians were tightly controlled. During all sessions both physicians followed a common script that provided guidelines with respect to the timing and type of interaction with the paramedic. Both physicians were equally available during each session, i.e., they were isolated from other activities in the hospital and received no interruptions during the session. Furthermore, both physicians participated in almost the same number of sessions in each condition as their scheduling allowed, i.e., one physician participated in 13 sessions in the $2 \mathrm{D}$ condition and 12 sessions in the 3D proxy condition.

We also examined how many paramedics knew the consulting physician from previous interactions. During interviews paramedics reported that in their everyday jobs how well they know individual physicians makes a difference with respect to levels of trust. In the 2D condition five paramedics had previously met the physician. When asked to identify how well they knew the physician on a scale of 1 (recognize his face) to 10 (know him very well), the average response was 5.2. In comparison, 6 paramedics in the 3D proxy condition had previously met the physician but the average response with respect to how well they knew the physician was 2.6. Thus one would expect levels of trust to be higher in the 2D condition based on the stronger existing relationships between paramedics and physicians. However all trust was higher in the 3D proxy condition.

\subsubsection{Postinterview results}

Trust between physicians and paramedics is important because it empowers paramedics. Trust from a physician increases a paramedic’s self-confidence, and self-confidence is required when performing medical tasks. As one emergency services manager explained:

If you don't have trust from the physician, you don't have confidence. And if you don't have confidence, you can't do these skills. ...You know, it's about... sixty percent knowledge and about forty percent confidence...It's really amazing... these two things have to mesh-otherwise, you won't have a successful skill or a [successful]outcome.

In the U.S. health care system physicians are considered the elite and paramedics are required by law to trust and follow physicians’ instructions, yet this trust may not be reciprocated. Paramedics explained:

The physicians are always considered the elite in the health care chain, and the paramedics are always the lowest. You have to bow under... [our medical doctor]... there's nothing we [paramedics] can really do... We all function under his [medical] license.

The majority of ER [emergency room] doctors...just don't listen to us. 


\section{A lot of times we get treated like dirt [by physicians.]}

We are ... struggling to be respected and trusted.

The hierarchical and legal aspects of the health care system (along with the use of a constructive interaction script and isolation of the physician from other activities during each session) may help explain the generally high responses for the trust questions in the post-questionnaire. Yet there are statistically significant differences with respect to perceptions of the collaborating physician’s competence, promise fulfillment, receptivity and overall trust.

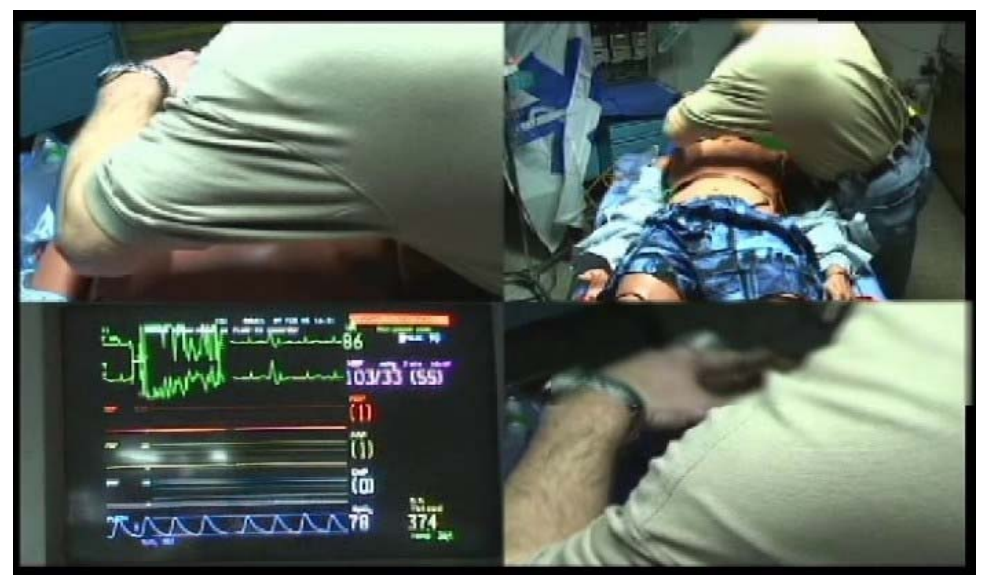

Figure 4. Typical remote physician's view in the 2D condition.

The higher responses may be attributed to physicians being able to more clearly see what the paramedic was doing and asking fewer 'dumb' or unhelpful questions. As illustrated in Figure 4 the remote physician's view in the 2D condition was periodically blocked by the paramedic and/or medical equipment used in the procedure, even though the cameras were optimally placed for this specific medical scenario. When a physician could not see what the paramedic was doing and the patient's condition was deteriorating, the paramedic could be asked to move and/or describe his actions, placing an additional burden on the paramedic. Paramedics commented:

[The physician] even asked me to move my hand at one time so he could actually see that I was in the right place...

[The physician] kept saying that my fingers kept getting in the way of his view...he couldn't see

[The physician] had to say...I can't see what you're doing.

These types of requests from physicians can be perceived by paramedics as frustrating and unhelpful. For example, when describing everyday collaboration with physicians paramedics said:

A good physician would let you perform and wait on you to talk...unless he sees something really, really stupid. 
Sometimes they're not familiar with our protocols. They're not familiar with what we have and you have to literally walk them through it...You know it gets frustrating sometimes.

Sometimes they don't see what we see...so sometimes they don't believe what you tell them.

Thus the ability to see details in the 3D proxy condition appears to reduce or eliminate the need for the physician to make requests for the paramedic to move or describe his or her actions in greater detail, subsequently increase the paramedics’ perceptions of the physician's competence, promise fulfillment and receptivity and overall trust between the paramedic and physician.

\section{Discussion}

Information studies and social informatics research (excluding information retrieval research) typically employs field study approaches, collecting and analyzing interviews, observations, diaries, artifacts and surveys. These approaches are not feasible when the information and communications technology under investigation does not yet exist. Yet it is useful to investigate the potential of new technology before it exists. Such investigations can provide new insights regarding positive and negative impacts of the technology on society, including ways to reduce or eliminate the negative impacts, and either justify or slow down large investments typically required to develop and deploy new technology. Our approach was to develop an experiment—a controlled lab study — that incorporated as much of the context and situation of interest as possible and simulated the proposed technology. It is not an approach without challenges. Resources are required to understand and recreate the target situation and context. It can be challenging to recruit and coordinate study participants whose skills and knowledge are in high demand elsewhere. Yet all research faces challenges and costs. Our approach provides insights regarding future technology before large resources are spent or harmful consequences from technology deployment occur.

Hypothesis $H 1$ that predicted paramedics would perceive the information provided by the collaborating physician more useful in the $3 \mathrm{D}$ proxy condition than in the $2 \mathrm{D}$ condition is supported by the data. All information provided by the physician, except that regarding intubation, was judged to be more useful in the 3D proxy condition. In addition to rating the usefulness lower, the paramedics in the 2D condition showed significantly greater variance in their responses. This variance might be related to previous work experience in the sense that less experienced paramedics perceived the information from the physician to be more useful than the more experienced ones. However, this correlation was not present in the 3D proxy condition. Usefulness of information is an important aspect of emergency medical care because receiving useful information has an impact not only on current task performance but also learning and future task performance. 
Hypothesis $\mathrm{H} 2$ that proposed paramedics would judge the quality of interaction with the physician higher in the 3D proxy condition is partially supported. The paramedics reported that their interaction was less constrained and better overall in the 3D proxy condition than in the 2D condition. Although the interaction in the 3D proxy condition was also judged to be good, more accurate and easier, these differences were not statistically significant. During interviews, paramedics in both conditions reported an initial awkwardness to their interaction with the physician. However, paramedics in the 2D condition mentioned many more difficulties. Overall, 75\% of the paramedics in the 2D condition mentioned problems interacting with the physician compared to only $25 \%$ of the paramedics in the 3D proxy condition. Future work includes analysis of the videos from both conditions to gain further insights regarding interaction among the paramedics and physicians.

Hypothesis $H 3$ predicted that paramedics would report higher levels of trust with respect to the consulting physician in the $3 \mathrm{D}$ proxy condition than in the 2D condition. There is partial support for this hypothesis. Competence, promise fulfillment and receptivity of the physician, as well as overall trust towards the physician were statistically higher in the 3D proxy condition. However, there was no statistically significant difference between conditions with respect to consistency, fairness, integrity, loyalty, discretion, openness and availability.

It appears that the increased depth perception and ability to dynamically change views are important features for 3D telepresence technology. We often saw physicians changing their viewpoint during the experiment sessions, bending down to get a side-angle view, as well as standing up on tiptoe and leaning over the victim. The physicians did not need to ask paramedics to move so they could see the patient better. The paramedic was free to focus on the medical task at hand, and did not need to worry about the physician's view.

However our mixed results may also reflect the potential negatives the technology might introduce to paramedics work. For example, the technology has the potential to make paramedics’ work visible and subsequently evaluated in new ways. Paramedics explained:

It was nice that [the physician] was there and he had your back and he was going to walk you through it. But then again it's kind of intimidating because you feel like you get trained to do this right...you're scared you might mess up, and they say, we want you trained better than this.

It kind of makes somebody nervous being monitored by a physician, someone of such higher training. And you're afraid to make a mistake because this person could be the person that ends up saying [whether] you get to do more, and where you work or not.

Ways to avoid these negative consequences that were mentioned by paramedics included opportunities for paramedics and physicians to get to know one another personally and professionally, open and non-judgmental communication 
practices, and increased understanding regarding joint responsibilities and priorities between paramedics in the field and physicians and nurses in the hospital.

The results overall illustrate that 3D telepresence technology shows some promise with respect to remote medical consultation in emergency medical situations. Yet it is not all good news; some questions remain regarding the value and implementation of 3D telepresence technology for use in emergency health care. These questions are further explored in the paper, "Exploring the potential of video technologies for collaboration in emergency medical care, Part II: Task Performance", and in a study under way exploring the wider context of emergency health care from the perspective of hospital administration, physicians, hospital IT managers, government health care policy, and health insurance providers.

\section{Acknowledgements}

Our sincere thanks to the study participants, Jim Mahaney who provided expert technical assistance, Chris Wiesen and Steve Pollack who provided suggestions regarding quantitative data analysis, Ann-Sofie Axelsson who provided comments on a draft, and the anonymous reviewers. This research is supported by the U.S. National Library of Medicine (National Institutes of Health), contract N01-LM-3-3514, 3D Telepresence for Medical Consultation: Extending Medical Expertise Throughout, Between and Beyond Hospitals.

\section{References}

Alem, L., Hansen, S., \& Li, J. (2006). Evaluating clinicians' experience in a telemedicine application: A presence perspective. In Proc. OZCHI '06 Conf., vol. 206, (pp. 46-54). NY: ACM Press.

American Society of Anesthesiologists Task Force on Difficult Airway Management. (2003). Practice guidelines for management of the difficult airway. Anesthesiology, 98, 1269-1277.

Bair, A.E., Panacek, E.A., Wisner, D.H., Bales, R., \& Sakes, J.C. (2003). Cricothyrotomy: A 5-year experience at one institution. The Journal of Emergency Medicine, 24(2), 151-156.

Bardram, J., Bossen, C., \& Thomsen, A. (2005). Designing for transformations in collaboration. In Proceedings of the GROUP’05 Conference (pp. 294-303). NY: ACM Press.

Bos, N., Olson, J., Gergle, D., Olson, G., \& Wright, Z. (2002). Confidence and trust: Effects of four computer-mediated communications channels on trust development. In Proceedings of the SIGCHI Conference on Human Factors in Computing Systems (pp. 135-140). NY: ACM Press. 
Bradner, E., \& Mark, G. (2001). Social presence with video and application sharing. In Proceedings of the GROUP '01 Conference (pp. 154-161). NY: ACM Press.

Butler, J.K. (1991). Toward understanding and measuring conditions of trust: Evolution of a conditions of trust inventory. Journal of Management, 17(3), 643-663.

Carroll, J.M., Rosson, M.B., Convertino, G., \& \& Ganoe, C.H. (2006). Awareness and teamwork in computer-supported collaborations. Interacting with Computers, 18, 21-46.

Chidambaram, L., \& Jones, B. (1993). Impact of communication medium and computer support on group perceptions and performance: A comparison of face-to-face and dispersed meetings. MIS Quarterly, 17(4), 465-491.

Clark, H. (1996). Using Language. Cambridge, UK: Cambridge University Press.

Coates, T.J., \& Goode, A. (2001). Towards improving prehospital trauma care. Lancet, 357(9274), 2070.

Cottrell, N., Wack, D., Sekerak, G., \& Rittle, R. (1968). Social facilitation of dominant responses by the presence of an audience and the mere presence of others. J. Pers. Soc. Psycho., 9(3), 245-250.

Dourish, P., Adler, A., Belloti, V., \& Henderson, A. (1996). Your place or mine? Learning from long-term use of audiovide communication. Computer Supported Cooperative Work, 5 (1), 33-62.

Elliot, J.M. (1999). Airway management. In I. Greaves \& K.M. Porter (Eds.), Pre-hospital Medicine (pp. 27-48). London: Arnold.

Field, M. (Ed.) (1996). Telemedicine: A guide to assessing telecommunications for health care. Washington, DC: National Academy Press.

Fussell, S. R., Setlock, L. D., \& Kraut, R. E. (2003). Effects of head-mounted and scene-oriented video systems on remote collaboration on physical tasks. In Proceedings of the CHI '03 Conference (pp. 513-520). NY: ACM Press, NY.

Gale, C. (1998). The effects of gaze awareness on dialog in a video-based collaborative manipulation task. Proceedings of CHI '98. (pp. 345-346). NY: ACM Press.

Gawande., A. (2001). When doctors make mistakes. In A. Gawande, Complications: A Surgeon's Notes on an Imperfect Science (pp. 47-74). NY: Henry Holt and Company.

Handy, C. (1995). Trust and the virtual organization. Harvard Business Review, May-June, 43-50.

Hauber, J., Regenbrecht, H., Billinghurst, M., \& Cockburn, A. (2006). Spatiality in videoconferencing: Trade-offs between efficiency and social presence. In Proceedings of the CSCW'06 Conference (pp. 413-422). NY: ACM Press. 
Höysniemi, J., Hämäläinen, P., \& Turkki, L. (2004). Wizard of Oz prototyping of computer vision based action games for children. In Proc. Conf. on Interaction Design and Children. NY: ACM Press.

Jarvenpaa, S., \& Leidner, D. (1999). Communication and trust in global virtual teams. Organization Science, 10(6), 791815.

Levin, D.Z., Cross, R., \& Abrams, L.C. (2002). Why should I trust you? Predictors of interpersonal trust in a knowledge transfer context. Presented at 2002 Academy of Management Meetings, Denver. CO. Retrieved October 29, 2007 from http://www.providersedge.com/docs/km_articles/Why_Should_I_Trust_You.pdf

Levin, D.Z., \& Cross, R. (2004). The strength of weak ties you can trust: The mediating role of trust in effective knowledge transfer. Management Science, 50(11), 1477-1490.

Li, J., Wilson, L., Stapleton, S., \& Cregan, P. (2006). Design of an advanced telemedicine system for emergency care. In Proc. OZCHI '06 Conf. (pp. 413-416). NY: ACM Press.

Mayers, R.C., \& Davis, J.H. (1999). The effect of the performance appraisal system on trust for management: A field quasiexperiment. Journal of Applied Psychology, 84(1), 123-136.

Mbarika, V. (2004). Is telemedicine the panacea for Sub-Saharan Africa's medical nightmare? CACM, 47(7), 21-24.

Meyer, A. (1998). Death and disability from injury: A global challenge. J Trauma, 44(1), 1-12.

Mun, S. (2000). Project Phoenix: Scrutinizing a telemedicine testbed. Final Project Report, National Library of Medicine, Contract \#N01-LM-6-3544.

Olson, G.M., \& Olson, J.S. (2000). Distance matters. Human-Computer Interaction, 15(2-3), 139-178.

Olson, J.S., \& Teasley, S. (1996). Groupware in the wild: Lessons learned from a year of virtual collocation, Proceedings of the ACM 1996 Conference on Computer Supported Cooperative Work (pp. 419-427). NY: ACM Press.

Orlikowski, W. (1993). Learning from Notes: Organizational issues in groupware implementation. The Information Society, 9(3), 237-252.

Robson, C. (2002). Real World Research. Cambridge, MA: Blackwell.

Rocco, E., Finholt, T., Hofer, E., \& Herbsleb, J. (2001). Out of sight, short of trust. Presentation at the Founding Conference of the European Academy of Management, Barcelona, Spain.

Rousseau, D., Sitkin, S., Burt, R., \& Camerer, C. (1998). Not so different after all: A cross-discipline view of trust. Academy of Management Review, 23(3), 393-404. 
Sellen, A. (1992). Speech patterns in video-mediated conversations. In Proceedings of the ACM CHI'92 Conference (pp. 49-59). NY: ACM Press.

Shadish, W., Cook, T., \& Campbell, D. (2002). Experimental and Quasi-experimental Designs for Generalized Causal Inference. NY: Houghton Mifflin.

Short, J., Williams, E., \& Christie, B. (1976). The Social Psychology of Telecommunications. NY: Wiley.

Smith, J.B., \& Barclay, D.W. (1997). The effects of organizational differences and trust on the effectiveness of selling partner relationships. Journal of Marketing, 61, 3-21.

Smith, B., \& Gaba, D. (2000). Simulators. In C. Lake, C. Blitt, \& R. Hines (Eds.) Clinical Monitoring: Practical Application. NY: WB Saunders Company. Preprint retrieved February 11, 2008 from http://simtech.stanford.edu/ "Docs" Sonnenwald, D.H. (1999). Evolving perspectives of human information behavior: Contexts, situations, social networks and information horizons. In T. D. Wilson \& D. K. Allen (Eds.), Exploring the Contexts of Information Behavior: Proceedings of the Second International Conference in Information Needs (pp. 176-190). London: Taylor Graham.

Sonnenwald, D.H., Maglaughlin, K.L., \& Whitton, M. C. (2004). Designing to support situational awareness across distances: An example from a scientific collaboratory. Information Processing and Management, 40(6), 989-1011.

Sonnenwald, D.H., Whitton, M.C., \& Maglaughlin, K.L. (2003). Evaluating a scientific collaboratory: Results of a controlled experiment. ACM Transactions on Computer Human Interaction, (10)2, 150-176.

Star, S.L., \& Ruhleder, K. (1996). Steps toward an ecology of infrastructure: Problems of design and access in large information systems. Information Systems Research, 7, 111-134.

Tachakra, S. (2001). Depth perception in telemedical consultations. Telemed J E-Health, 7 (2), 77-85.

Thompson, N.J., Raftery, J., \& Thompson, K.E,. (2003). Client conditions of trust within virtual organisations: an application of Butler’s conditions of trust inventory in the UK construction sector. Retrieved June 16, 2004 from http://www.mubs.mdx.ac.uk/Research/Discussion_Papers/Marketing/dpap\%20marketing\%20no.20.pdf

Vaughan, Liwen (2001). Statistical Methods for the Information Professional. Medford, N.J.: Information Today

Welch, G., Sonnenwald, D.H., Mayer-Patel, K., Yang, R., State, A., Towles, H., Cairns, B., \& Fuchs, H. (2005). Remote 3D medical consultation. Second International Conference on Broadband Networks, Vol. 2, (pp. 1026-1033). NY: IEEE Press. 